\title{
Contrast-enhanced ultrasound of the pediatric bowel
}

\author{
Ami Gokli ${ }^{1}$ (D) Jonathan R. Dillman ${ }^{2} \cdot$ Paul D. Humphries ${ }^{3} \cdot$ Damjana Ključevšek $^{4} \cdot$ Hans-Joachim Mentzel $^{5}$. \\ Erika Rubesova ${ }^{6}$. Marcelo S. Takahashi ${ }^{7}$. Sudha A. Anupindi ${ }^{1}$
}

Received: 11 June 2020 /Revised: 13 August 2020 / Accepted: 30 September 2020 / Published online: 12 May 2021

(C) Springer-Verlag GmbH Germany, part of Springer Nature 2021

\begin{abstract}
Contrast-enhanced ultrasound (CEUS) has emerged as a valuable modality for bowel imaging in adults and children. CEUS enables visualization of the perfusion of the bowel wall and of the associated mesentery in healthy and disease states. In addition, CEUS images can be used to make quantitative measurements of contrast kinetics, allowing for objective assessment of bowel wall enhancement. Bowel CEUS is commonly applied to evaluate inflammatory bowel disease and to monitor treatment response. It has also been applied to evaluate necrotizing enterocolitis, intussusception, appendicitis and epiploic appendagitis, although experience with these applications is more limited. In this review article, we present the current experience using CEUS to evaluate the pediatric bowel with emphasis on inflammatory bowel disease, extrapolating the established experience from adult studies. We also discuss emerging applications of CEUS as an adjunct or problem-solving tool for evaluating bowel perfusion.
\end{abstract}

Keywords Bowel · Children · Contrast-enhanced ultrasound · Crohn disease · Inflammatory bowel disease $\cdot$ Ultrasound · Ultrasound contrast agents

\section{Introduction}

Imaging plays an integral part in the evaluation of bowel pathology and is performed for various congenital, inflammatory and neoplastic conditions in children. It can provide prompt and accurate diagnosis and guide further management decisions, ensuring optimal care.

Electronic supplementary material The online version of this article (https://doi.org/10.1007/s00247-020-04868-x) contains supplementary material, which is available to authorized users.

Ami Gokli

agokli@northwell.edu

1 Department of Radiology,

Children's Hospital of Philadelphia,

Perelman School of Medicine, University of Pennsylvania,

3401 Civic Center Blvd.,

Philadelphia, PA 19104, USA

2 Department of Radiology,

Cincinnati Children's Hospital Medical Center,

University of Cincinnati College of Medicine,

Cincinnati, OH, USA

3 University College London Hospital NHS Trust, Great Ormond Street Hospital for Children,

London, UK
Conventionally, MRI or CT is the more common firstline imaging examinations performed to visualize the entirety of the bowel and evaluate for extramural complications. High-resolution gray-scale US coupled with color and power Doppler techniques holds value as a complementary examination for most inflammatory or perfusional bowel pathology.
4 Department of Radiology, University Children's Hospital Ljubljana, Ljubljana, Slovenia

5 Section of Pediatric Radiology, Institute of Diagnostic and Interventional Radiology, University Hospital Jena, Jena, Germany

6 Department of Radiology, Lucile Packard Children's Hospital, Stanford University Medical Center, Stanford, CA, USA

7 Pediatric Radiology Department, Diagnósticos da America (DASA), São Paulo, Brazil 
Contrast-enhanced ultrasound (CEUS) has emerged as an important radiation-free imaging modality with an expanding range of applications in bowel imaging [1-12]. When evaluating bowel wall inflammation or perfusion status, CEUS is performed with intravenous administration of ultrasound contrast agents (UCAs). UCAs are purely intravascular agents and do not diffuse into the interstitial compartment, allowing for identification of blood flow within the vascular network in normal and pathological tissues. In people with inflammatory bowel disease (IBD), intravenous CEUS can improve the diagnostic sensitivity of Doppler US to visualize bowel wall and mesenteric perfusion in real time [1-3]. It can also be useful for evaluating the severity of the disease, monitoring response to therapy, and assessing for complications or disease relapse [1-5, 12-19]. Quantitative CEUS with analysis of timeintensity curves can provide measurable information regarding the magnitude and rapidity of perfusion in the bowel wall and associated mesentery and objectively evaluate treatment response in people with IBD [3,13-16, 18-24]. When assessing extramural complications, intravenous CEUS can delineate inflammatory reaction around the bowel lumen and differentiate between inflammatory mass (phlegmon) and abscess [25]. In addition, diluted solutions of UCA/normal saline can be instilled through tubes and drains into abscesses or fluid collections to confirm the correct position of the drains and to assess for possible communications or fistulous tracts (i.e. intracavitary CEUS applications) [26, 27].

There is widespread cumulative experience from the use of CEUS for evaluating inflammatory bowel pathology in adults [1-8, 13-25]. However, CEUS applications for evaluating IBD in children are limited [9-12]. CEUS might also play a role when knowledge of bowel perfusion can help direct care in children with other bowel-related conditions, such as necrotizing enterocolitis, intussusception, appendicitis, epiploic appendagitis, vasculitis or other miscellaneous causes of hypo- or hyperperfusion $[7,8,10,28]$. These applications for CEUS are increasingly being explored.

In the present article, we review the current experience with bowel CEUS, predominantly for evaluating IBD in adults and children, with an emphasis on the examination technique and interpretation of imaging findings. We also discuss other applications for bowel CEUS, particularly as an adjunct or problem-solving tool, and we highlight evolving uses.

\section{Imaging technique for bowel contrast-enhanced ultrasound}

\section{Patient preparation}

Children are asked to refrain from solid foods for $4 \mathrm{~h}$ prior to their appointment. The reasoning behind this recommendation is to avoid enteric content limiting adequate bowel visualization and to prevent potential post-meal increase of blood flow to the bowel wall that could result in variable follow-up examination. However, for urgent same-day examinations in young children and infants, no patient preparation is required prior to CEUS. Yet, children are encouraged to drink non-carbonated fluids prior to the examination to displace bowel gas with fluid, which improves visualization. This can also help to better visualize a prestenotic bowel dilation if present.

\section{Pre-contrast ultrasound scan}

Conventional sonographic evaluation of the bowel encompasses standard gray-scale and color/power Doppler US prior to CEUS. All bowel segments are examined in a systematic approach starting with the terminal ileum and then going over to the cecum, ascending colon, transverse colon, descending colon, sigmoid colon and rectum, followed by assessment of the small bowel in all four quadrants. This enables the examiner to orient to the anatomy and assess the entirety of the bowel and other intra-abdominal contents. With a baseline pre-contrast US, the examiner can identify the most abnormal loop of bowel for the subsequent contrast study. The selected bowel loop should be adequately and consistently visible, have little to no peristalsis, and preferably be located far from the diaphragm to minimize respiratory motion. To mitigate peristalsis, some examiners recommend administering antispasmolytics prior to CEUS exams. Pre-contrast US assesses the diseased bowel location, total length, wall thickness, morphology of the bowel wall, and vascularity using color/power Doppler US [29, 30]. Other imaging features examined on baseline US include echogenicity and vascularity of the mesenteric fat, presence of enlarged lymph nodes, and complications such as inflammatory mass, abscesses, strictures and fistulae.

\section{Contrast-enhanced ultrasound imaging technique}

When performing CEUS in children, high-resolution linear transducers in the frequency range of $5-15 \mathrm{MHz}$ are most commonly used with contrast-specific imaging techniques for qualitative and quantitative assessment. A lowerfrequency linear transducer in the range of $2-12 \mathrm{MHz}$ is reserved for obese patients and bowel segments located deeper in the abdomen or pelvis; however, visualization might be limited by depth. A wide-band convex transducer, such as a $1-5-\mathrm{MHz}$ or $2-9-\mathrm{MHz}$ transducer (a wider range of other available transducers might suffice) can also be used to better visualize long segments of inflamed bowel.

Contrast imaging technical parameters include a low mechanical index and contrast-specific mode with signal subtraction, whereby the echoes from the background tissue are suppressed so that a microbubble-only image is generated. The focal zone is set at the maximal depth. A dual screen display is commonly used, on which the US screen is split with a gray- 
scale anatomical reference image alongside the contrast image.

\section{Ultrasound contrast agent dose and administration technique}

Lumason (Bracco Diagnostics, Monroe Township, NJ) is the United States Food and Drug Administration (FDA)-approved UCA for pediatric intravenous liver and cardiac as well as intravesical applications. Lumason is marketed as SonoVue (Bracco Imaging, Milan, Italy) outside the United States. The approved dose of Lumason to evaluate the liver is $0.03 \mathrm{~mL} / \mathrm{kg}$ up to a maximum of $2.4 \mathrm{~mL}$ per single injection. For bowel CEUS in adults or children, there are no FDA dose recommendations. The use of UCA for bowel imaging is off-label.

Several studies in adults have shown that a higher volume of UCA is required for CEUS imaging of the bowel compared to solid parenchymal organs because bowel CEUS is performed using higher-frequency linear transducers; single UCA doses of $2.4 \mathrm{~mL}, 4.8 \mathrm{~mL}$ and $5 \mathrm{~mL}$ have been reported [3, 14, 31, 32].

In children, the studies conducted with Lumason for evaluating children with Crohn disease used the $0.03 \mathrm{~mL} / \mathrm{kg}$ weight-based dose, the same as the approved dose for pediatric liver and cardiac CEUS applications [9, 11]. A previous study with the use of SonoVue in children with Crohn disease calculated the UCA dose according to the formula: dose $(\mathrm{mL})$ $=$ child age (years) $/ 10$, with a minimum $0.1 \mathrm{~mL}$ per single injection [12]. In another study, which included premature infants ranging in age from 1 day to 80 days at the time of CEUS, the reported total dose of Lumason for evaluating bowel perfusion was $0.4-0.6 \mathrm{~mL}$ [10].

Both bolus injection and infusion techniques have been reported for UCA administration during bowel CEUS. Most studies in adults have used a bolus administration, for which a single volume of UCA is injected at a rate of approximately $1 \mathrm{~mL} / \mathrm{s}$ through the $180^{\circ}$ (in-line) port of a 3-way stopcock followed by a normal saline flush from the $90^{\circ}$ (side) port of the stopcock [2, 14, 15, 22, 24, 32-39]. In 2017, a pictorial essay was published about the feasibility of using a CEUS destruction-replenishment method in adults with Crohn disease [17]. This method requires delivery of contrast agent at a steady flow rate within the blood circulation, which can be achieved using dedicated infusion pumps. In this study, continuous intravenous infusion of UCA was performed at a $1.5-\mathrm{mL} / \mathrm{min}$ infusion rate. Analysis of contrast kinetics within the target tissue was performed following destruction of contrast microbubbles by briefly increasing the mechanical index (flash mode). Recently, another study used consecutive administration of a bolus injection followed by a continuous drip infusion in the same session to evaluate a group of people with Crohn disease [13]. The bolus injection was performed with $0.2 \mathrm{~mL}$ Definity (Lantheus Medical Imaging, North Billerica, MA) followed by $10 \mathrm{~mL}$ of normal saline flush. The subsequent drip infusion consisted of $1.6 \mathrm{~mL}$ of Definity diluted in $50 \mathrm{~mL}$ of normal saline and administered at a fixed rate of $5 \mathrm{~mL} / \mathrm{min}$. No dedicated pump was used in this study. Multiple sequential 30-s cine loops were obtained after transiently disrupting the UCA by increasing the mechanical index briefly (flashing at 3 frames/s).

\section{Image acquisition: Qualitative bowel contrast- enhanced ultrasound}

Qualitative bowel CEUS is used to assess the degree and pattern of transmural and mesenteric enhancement. Following intravenous UCA administration, a continuous video clip acquisition of the selected bowel segment is performed, starting at the time of UCA injection and continuing for approximately $2 \mathrm{~min}$. For qualitative evaluations, the majority of studies have been performed with bolus intravenous injection [18, 20, 39-44]. Imaging in the long axis of the bowel is typically done, with the transducer held in a stationary position over the examined bowel loop. Children are instructed to have normal shallow breathing throughout the CEUS exam.

Bowel enhancement occurs in three consecutive phases: (1) initial phase (0-20 s after UCA injection), during which contrast agent is not usually visible within the bowel wall; (2) enhancing or enteric phase (20-30 s after contrast injection), which depicts most of the UCA within the bowel wall vasculature; and (3) late phase (30-60 s), which involves variable persistent enhancement or washout, depending on the bowel wall state [45]. Normal bowel enhancement typically occurs in a homogeneous transmural pattern with a low level of enhancement early on, variable enhancement at 20-30 s, and washout of contrast agent after 30 s [45] (Online Supplementary Material 1). Actively inflamed bowel generally shows similar findings on CEUS as seen on CT and MR enterography, including rapid and intense transmural enhancement leading to a high peak intensity and then to slow contrast washout during the late phase (Online Supplementary Material 2). Less often, only enhancement of the inner mucosal/submucosal layer is seen $[1,42]$.

\section{Image acquisition: Quantitative bowel contrast- enhanced ultrasound}

Quantitative analysis is used to evaluate the UCA transit time through the examined tissue and to assess the speed of contrast wash-in and washout [46]. To quantitate bowel wall perfusion with CEUS, time-intensity curves are generated by calculating the blood flow parameters within regions of interest (ROIs) that are manually selected and placed within the abnormal bowel segment. A longitudinal plane is preferred to minimize out-of-plane effects from respiratory motion and to include an adequately long segment of bowel in the field-of-view [1]. A manually selected ROI is drawn along the thickened bowel wall. The size of the ROI influences the results of the time- 
intensity curve, particularly the peak enhancement, and thus the size of the ROI must be standardized. This is of great importance for follow-up comparisons. A recent metaanalysis described varying normal bowel wall thickness for different segments of bowel wall [47]. The bowel wall is generally considered to be abnormally thickened when it is greater than $3 \mathrm{~mm}$ for the small bowel and colon; a wall thickness of at least $3 \mathrm{~mm}$ accommodates placement of the ROI [48].

A continuous video clip acquisition is initiated at the moment of UCA injection or infusion and lasts for approximately 2 min $[1,3]$. Standardization of the length of the cine clip allows for comparison of perfusion measurements between studies. The data from the 2-min video clip are stored on the US system and then analyzed with specific software that is integrated into the same US unit or at an independent workstation with third-party software. Advantages of third-party software include the ability to compare studies across different machines and vendors and to afford more advanced processing/parametric maps.

The imaging parameters derived from time-intensity curves analysis include peak enhancement, time to peak, rate of contrast wash-in/washout (slope), and the area under the curve that corresponds to regional blood flow and volume [1, 13, $19,20,22,23]$. All values are calculated in arbitrary intensity units and can be presented in a linear scale or converted to a logarithmic scale. Additionally, quantitative CEUS can generate parametric maps based on color scales of signal intensities, such that hyperemic bowel segments are represented, for example, by the color red and segments with normal bowel wall vascularity by the color blue $[15,23,49]$.

Compared to qualitative CEUS, quantitative CEUS allows for a more precise assessment of bowel wall enhancement that is not affected by subjective, observer-related, visual assessment. The objective nature of quantification has been found to be useful for monitoring treatment response $[16,19,36]$. It is important to note that follow-up examinations must be performed under the same conditions because many factors affect the time-intensity curves analysis, including fasting state, contrast dose, US machine settings, clip duration and size of ROI. Table 1 summarizes the CEUS examination protocol for pediatric bowel imaging.

\section{Evaluation for inflammatory bowel disease}

Inflammatory bowel disease (IBD) is characterized by chronic, relapsing and remitting intestinal inflammation that is typically classified into Crohn disease and ulcerative colitis [50]. Crohn disease can occur anywhere throughout the gastrointestinal tract from the mouth to anus, but most commonly it involves the terminal ileum and colon. It is discontinuous, transmural inflammation with stricturing or penetrating (fistulizing)

Table 1 Contrast-enhanced ultrasound (CEUS) examination protocol

Baseline gray-scale and color/power Doppler ultrasound evaluation

High-resolution linear transducers (7-14 MHz)

1. Targeted scan of the small bowel and colon to define disease location and extent

2. Selection of the most diseased segment of the bowel

3. Preparation for contrast-enhanced ultrasound (CEUS)

High-resolution linear probes (5-15 MHz or 2-12 MHz) or curved probes (1-5 MHz or 2-9 MHz) reserved for obese children and deeply located long segments

1. Contrast-specific mode settings

2. Low mechanical index

a. Focal zone at maximum depth

b. Optimize gain and dynamic range for examined segment of bowel before contrast administration

c. Document the selected bowel segment before contrast administration in contrast-specific mode (dual display) for baseline comparison

3. Patient in comfortable supine position with peripheral IV and 3-way stopcock

4. Contrast-enhanced ultrasound (CEUS)

Bowel loop of interest imaged in longitudinal axis to the bowel to reduce the effect of motion from breathing

1. Dual screen display (contrast and gray-scale modes side-by-side)

2. Ultrasound contrast agent is injected through the $180^{\circ}$ port of the 3 -way stopcock and saline flush through the $90^{\circ}$ port

3. Start the timer and begin video-recording when contrast injection is complete and before saline flush is ended or when the first bubbles are verified. Suggested video clip duration: 2 min

4. During initial continuous acquisition, the transducer is kept still and focused on the same interrogated loop of bowel to optimize quantification. Delayed imaging can be performed with intermittent acquisition of video clips or static images to reduce disruption of the microbubbles

5. Bowel evaluation and recording must be standardized to compare data among patients and between patient visits

$I V$ intravenous line 
features or a combination phenotype. Ulcerative colitis is characterized by diffuse and continuous inflammation of the colonic and rectal mucosa, with potentially mild inflammation of the ileum known as "backwash" ileitis [50, 51].

During an initial evaluation for IBD, children often undergo a series of imaging examinations, laboratory tests, and endoscopy with biopsies. US is traditionally used to evaluate wall thickening and hyperemia of the diseased bowel and to identify extramural complications. Motion artifact from bowel peristalsis or breathing movements can cause a generalized Doppler shift and lead to an overestimation of vessel density.

Over the last several years, MR enterography has become the imaging choice for evaluating children with IBD in many pediatric institutions. It enables accurate evaluation of the extent and severity of the disease and assessment of associated complications, including phlegmon or abscesses, strictures and fistulae. CT enterography is another imaging modality that can be used for bowel imaging. However, it is associated with cumulative risks of radiation exposure, and for that reason it is commonly reserved for those cases when MR enterography is not readily available or it is difficult to perform (e.g., need for oral contrast and anesthesia in a young child; need for acute bowel pathologies). In this setting, CEUS can be used as an alternative or complementary imaging tool to other cross-sectional modalities to accurately assess the state of the bowel wall and pericolonic tissues [1, 2, 43].

\section{Contrast-enhanced ultrasound for adults with inflammatory bowel disease}

The first studies on using CEUS to evaluate people with IBD were published in the early 2000s. These early studies demonstrated the feasibility and diagnostic efficacy of CEUS for IBD, and showed that its use could improve detection of abnormal bowel wall perfusion compared to color/power Doppler techniques. Since then, several studies comparing CEUS findings with clinical symptoms, laboratory tests, other imaging modalities, endoscopic indices, surgical findings, histopathology, and follow-up imaging have validated its diagnostic accuracy [35, 37-39, 52].

\section{Adult qualitative bowel contrast-enhanced ultra- sound studies}

The initial studies used visual imaging criteria to evaluate the degree and pattern of enhancement of the bowel wall before and after UCA administration [39, 42, 44]. Visual qualitative assessment determines the presence and severity of inflammation in the bowel wall and associated mesentery. Generally, when the bowel is inflamed, CEUS demonstrates rapid and more abundant transmural enhancement of the bowel wall than the adjacent tissues [1]. Bowel inflammation changes can be subjectively classified as mild, moderate or severe based on the observation of the enhancement pattern. Mild disease demonstrates low peak intensity and faster washout whereas more severe disease shows higher peak intensity and longer duration of enhancement effect [1]. Inflammatory involvement of the adjacent mesentery can also be characterized by evaluating the blood flow within the mesenteric vessels. The comb sign, referring to prominent hypervascularity (i.e., rapid and intense influx of contrast agent within the prominent linear vascular branches on the mesenteric side of the affected bowel), is not only depicted on MR enterography and CT enterography but also on CEUS $[1,43]$. CEUS reliably detects active Crohn disease as intense bowel wall enhancement. More important, imaging findings of active inflammation on CEUS led to a modified therapeutic approach in up to $65 \%$ of people who had negative clinical/laboratory tests [44].

\section{Adult semi-quantitative bowel contrast-enhanced ul- trasound studies}

In addition to qualitative analysis, early studies used various semi-quantitative methods to assess bowel wall perfusion following UCA injection, including measurement of the number of color foci indicative of blood vessels in the thickened bowel wall, measurement of the Doppler signal intensity, and assessment of the wall brightness post-UCA injection [38, 43, 53]. One study using semi-quantitative methodology analyzed the enhancement pattern of the bowel wall layers and compared the maximum thickness of the enhanced layer to the thickness of the entire bowel wall. Complete bowel wall enhancement and a layered enhancement pattern, with a thickened inner layer with hyperenhancement (increased blood flow), was found to be highly sensitive (81\%) and specific (57-63\%) for active Crohn disease [42].

\section{Adult quantitative bowel contrast-enhanced studies}

\section{Bowel inflammation}

With the advent of dedicated contrast software, quantitative analysis of contrast kinetics is now possible and can be employed to overcome the shortcomings of visual, qualitative and semi-quantitative CEUS analyses within the inflamed bowel wall. A number of studies have compared the perfusion parameters derived from time intensity curve analysis to different reference standards, including cross-sectional imaging, clinical, laboratory, endoscopic, surgical and histopathological scores [19, 35, 37, 38, 44, 49, 52, 54]. However, in these series inconsistent results were reported depending on the study design and reference method used.

When CEUS time-intensity curve analysis was compared to color Doppler US, it provided better detection of mural hyperenhancement in active Crohn disease. In addition, the 
quantitative bowel CEUS approach showed that people with Crohn disease had higher maximum enhancement of the inflamed bowel segment that persisted for a longer period of time compared to healthy controls who had lower and shorter duration of bowel wall enhancement [55].

Compared with operative macroscopic findings and histopathological scores, the CEUS time to peak had the strongest negative correlation with histological results: the shorter the time to peak, the higher the histopathological disease activity score. In addition, the shorter the time to peak, the higher the histological findings of acute inflammatory activity, such as erosions, excess of intraepithelial neutrophils, crypt distortion and crypt atrophy. On the contrary, time-to-peak values increased when clinical signs of inflammatory activity improved [54]. Another study that compared quantitative CEUS parameters with vascular density in biopsy samples found a higher enhancement peak, a shorter time to peak, and a higher regional blood flow and volume in people with high vascular density compared to those with low vascular density. In this study, the cutoff values to distinguish between active and inactive disease were identified for the percentage (\%) of increased enhancement after contrast administration $(>40.5 \%)$, and regional blood flow ( $>54.8 \mathrm{~mL} / \mathrm{min}$ ) [22].

However, findings differed when time to peak was correlated with endoscopy results [24]. One study did not find any significant correlation between the time to peak and the endoscopic indices. This might be because the endoscopist can only visualize the mucosal surface and not the entire bowel wall, referred to as endoscopic skipping [56]. In addition, the intestinal vascular remodeling that occurs in IBD is characterized by formation of stenotic neo microvessels and thus impaired mucosal perfusion [24]. According to another study, the imaging parameters that most correlate with the endoscopic findings of Crohn disease severity are the maximum intensity of the interior layer of the bowel wall and the maximum intensity of the entire bowel wall [20].

\section{Medical treatment response}

Contrast-enhanced ultrasound is also useful to evaluate for disease persistence that presents with continued mucosal inflammation despite clinical symptom relief. As highly effective biologic therapies have been introduced, treatment goals have shifted from managing symptoms to controlling mucosal inflammation so as to prevent further bowel damage [16, 19, 36]. To monitor the effectiveness of these treatments, inflammatory activity must be accurately assessed throughout the course of the therapy $[13,16,19]$. In addition, because of the chronic and progressive nature of the disease, repeat imaging evaluation is essential. CEUS can be performed repeatedly without additional risks.

When time-intensity curve parameters were compared with endoscopic and biopsy findings as well as clinical assessment in people with Crohn disease, a decreasing area under the timeintensity curve was found to be the most useful imaging parameter to discriminate responders from non-responders over the course of the pharmacologic treatment [19]. Another study found a significant decrease in time to peak after medical treatment that correlated with a decrease in the clinical disease index and laboratory values post-treatment [24].

When quantitative time-intensity curve analysis was coupled with the parametric color-coded maps with schematic representation of perfusion properties, the upward slope of the time-intensity curve and the area under the curve were found to be significantly lower after effective treatment and to correlate with improved clinical index scores [49]. According to this study, peak enhancement and the area under the curve are the most reliable indices to evaluate the success of the pharmacologic treatment.

\section{Extramural complications}

Extramural complications related to IBD include inflammatory mass and abscess, which appear on gray-scale US as a hypoechoic mesenteric lesion [25]. Inflammatory mass, previously called "phlegmon," refers to an ill-defined area of softtissue inflammation that typically develops at the site of a contained hollow viscous perforation, with no identifiable wall and some degree of internal blood flow on color/power Doppler US. On the other hand, abscess typically appears as a hypoechoic fluid collection with an irregular yet defined wall and a degree of peripheral blood flow on color/power Doppler. An inflammatory mass can occur with or without an associated abscess. CEUS enables examiners to confidently differentiate between these two extramural complications by either confirming intralesional enhancement in cases of an inflammatory mass or absence of intralesional enhancement in cases of an abscess. Differentiating between these two entities can have important management implications because an abscess might require drainage whereas an inflammatory mass usually responds to medical treatment [25].

\section{Strictures}

Contrast-enhance ultrasound has also been used to differentiate between inflammatory and fibrotic strictures, but a consensus regarding its utility has not been reached. Both conditions appear on US as increased bowel wall thickness. In a recently conducted animal study, imaging parameters derived from time-intensity curve analysis were compared to histopathological scores of tissue samples obtained from thickened bowel wall segments with experimentally induced inflammation mixed with variable degrees of fibrosis. In this study, quantitative CEUS perfusion parameters failed to differentiate bowel wall fibrosis if significant inflammation coexisted [57]. 
Although experimental evidence suggests that the enhancement characteristics of inflammation and fibrosis are difficult to distinguish because inflammation and fibrosis co-exist in the same segment of bowel, two clinical studies in adults with Crohn disease showed that quantitative CEUS differentiated fibrotic strictures from inflamed bowel segments [21, 52].

Given the fact that fibrotic lesions are associated with reduced blood flow and blood volume, the area under the curve and the percentage of maximal enhancement were the most useful imaging parameters for differentiating these two lesions. On the contrary, time to peak did not provide any guidance in this setting [52]. Despite what these authors have shown, there is no consensus on the CEUS quantification criteria to differentiate fibrosis and inflammation.

\section{Postoperative disease recurrence}

Postoperative disease recurrence can be detected in people with Crohn disease by using CEUS. One study showed that if CEUS performance at the anastomotic site reveals wall thickness $\geq 3 \mathrm{~mm}$ and bowel wall contrast enhancement value $\geq 46 \%$ (an arbitrarily defined threshold), the diagnostic accuracy for Crohn disease recurrence was $90.7 \%$ compared to endoscopically detected recurrence [14]. In the same study, the combination of bowel wall thickness $\geq 6 \mathrm{~mm}$ or $5-6 \mathrm{~mm}$ and contrast enhancement values $\geq 70 \%$ had a sensitivity of $90.3 \%$, specificity of $87 \%$, and accuracy of $88.9 \%$ to predict severe recurrent disease [14].

\section{Diagnostic accuracy}

Two meta-analyses were conducted to assess the performance of CEUS in the detection of active Crohn disease. A 2015 meta-analysis including 8 studies and 428 adults revealed that CEUS had a pooled sensitivity and specificity for active Crohn disease of $93 \%$ (95\% confidence interval [CI], 0.89-0.95) and 87\% (95\% CI, 0.81-0.91), respectively, using endoscopy/biopsy or clinical disease index as the reference standard [5]. In 2016, a second meta-analysis was conducted, encompassing 8 studies and 332 patients; 5 of the 8 included studies were the same as in the previous 2015 meta-analysis. The 2016 meta-analysis found that CEUS had a pooled $94 \%$ sensitivity (95\% CI, $0.87-0.97)$ and $79 \%$ specificity $(95 \% \mathrm{CI}$, 0.67-0.88); however, the authors noted that the studies had significant heterogeneity regarding CEUS methodology and definitions of enhancement thresholds for Crohn disease [4]. According to the subgroup analysis, relative bowel wall enhancement had the highest diagnostic value. Interestingly, time-intensity curve analysis for bowel wall perfusion using dedicated software was found to be less useful.

\section{Contrast-enhanced ultrasound for inflammatory bowel disease in children and adolescents}

Epidemiological data indicate that the incidence of IBD is rising worldwide, with up to $20-30 \%$ of IBD cases presenting during childhood [58]. Most children with IBD are diagnosed between 11 years and 16 years of age, but IBD can occur earlier, with a specific subset of pediatric IBD known as very early onset IBD occurring in children younger than 6 years [58-62]. Pediatric patients commonly present with severe and extensive disease, leading to significant morbidity, including delayed growth and nutritional impairment.

Similar to adults, active bowel inflammation in pediatric IBD has a characteristic imaging appearance that can be identified on CEUS (Fig. 1). When the bowel is actively inflamed, the wall enhances more rapidly with a faster time to peak. Mural enhancement is not only faster but also has a higher peak enhancement than normal bowel loops. In addition, the actively inflamed bowel retains contrast agent for a longer period of time, which is demonstrated by an increase in the area under the curve. Although no pediatric peak enhancement cut-off values exist, quantitative analysis can be guided by adult findings using peak enhancement cut-off values for mild, moderate and severe inflammation (Fig. 2) [11, 12].

Bowel CEUS indications in children with IBD are similar to those for adults, and CEUS is mainly used for the initial evaluation and follow-up of the disease. Time-intensity curve analysis is often used to monitor response to treatment and differentiate children who are responding to pharmacologic therapy from those who are not (Figs. 3, 4 and 5). Three studies with bowel CEUS including a pediatric population with IBD have been conducted to date. One, a review article published in 2016, presented experience from using quantitative CEUS analysis in pediatric patients with Crohn disease and highlighted its potential to become an integral part of the sonographic evaluation of IBD in children as well as adults [12]. The other, published in 2019, presented a case series of two adolescents and one young adult (age range: 16-20 years) with Crohn disease, all of whom underwent CEUS, shearwave elastography and MR enterography [11]. The first patient in the study had active inflammation of the terminal ileum, and CEUS accurately identified avid enhancement with more prompt wash-in, higher peak intensity, and a higher area under the curve compared to an adjacent, normal jejunal bowel loop. The second patient had a fibrotic ileocecal surgical anastomosis. In this case CEUS demonstrated minimal enhancement of the surgical anastomosis and mild enhancement of the more proximal ileum, suggestive of a fibrotic component of the former. CEUS findings were in agreement with subsequent MR enterography. The third patient had an ileocecal stricture, and CEUS of the terminal ileum demonstrated minimal contrast enhancement, suggesting no acute 
Fig. 1 Crohn disease and sigmoid wall thickening on baseline US examination in an 11-year-old girl. Contrast-enhanced ultrasound (CEUS) examination of the bowel. Dual image with simultaneous side-by-side display of the contrast-enhanced (left) and gray-scale (right) modes. Longitudinal still image captured from a CEUS video clip demonstrates significant mural thickening (arrowheads) with transmural hyperenhancement and marked enhancement of the surrounding mesentery (arrows), consistent with active disease

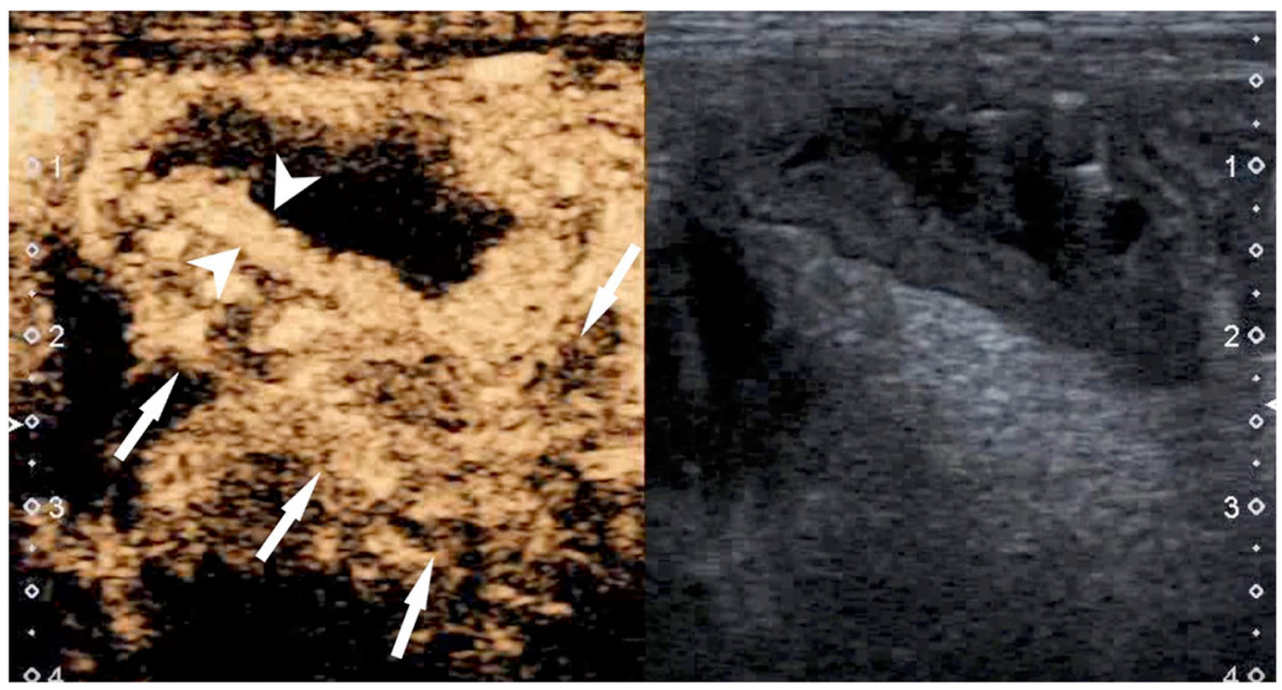

inflammation, findings that were in agreement with the shearwave elastography denoting increased bowel wall stiffness. The patient's CEUS findings were consistent with histological findings from a subsequent elective ileocecectomy that confirmed active chronic inflammation with stricture formation. Although this case series was small, the findings support the

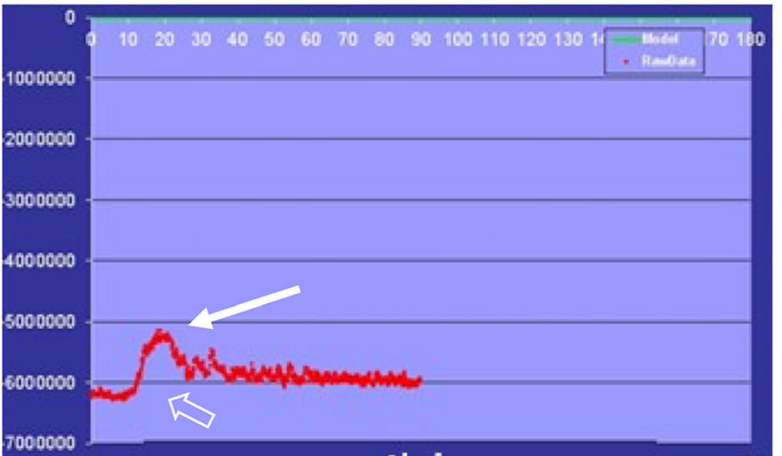

a

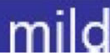

[s] b
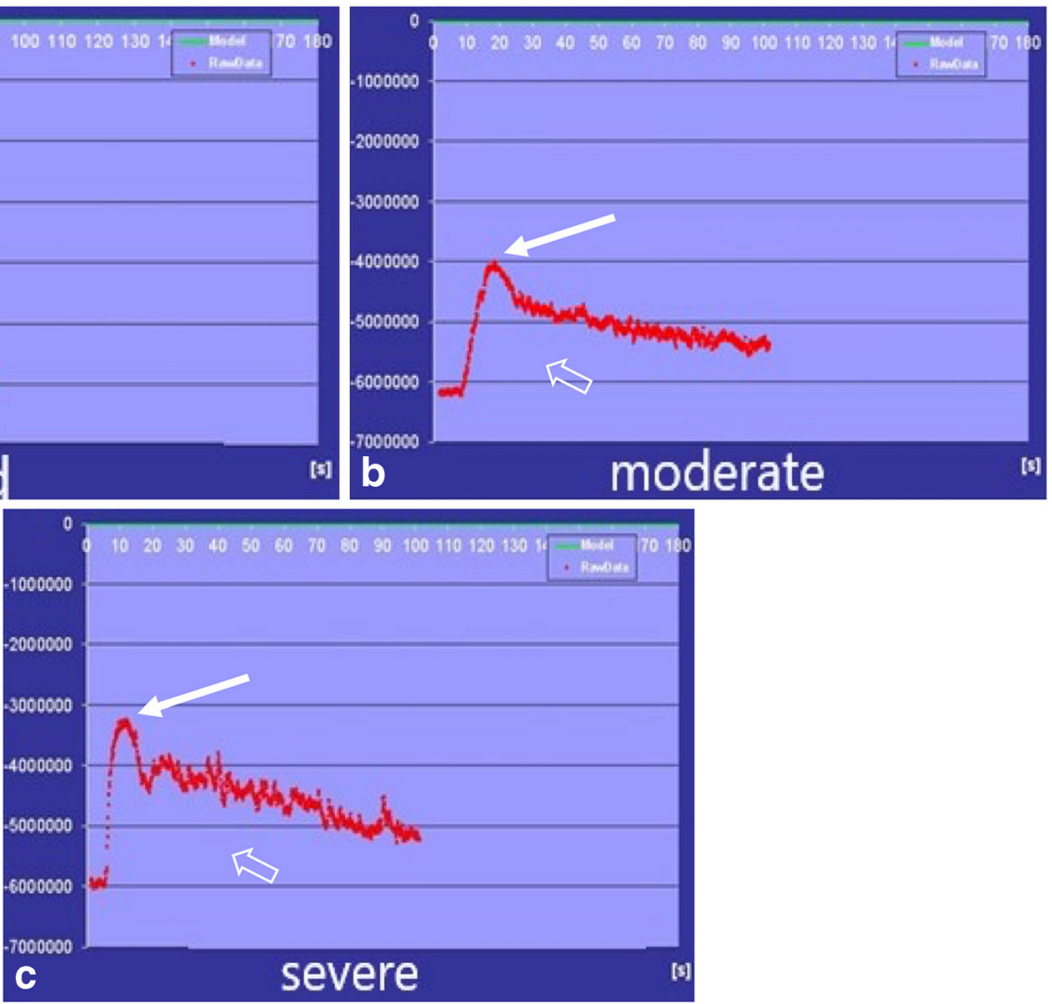

Fig. 2 Quantitative contrast-enhanced ultrasound (CEUS). a-c Timeintensity curves from three children with Crohn disease and variable severity of inflammatory bowel changes: a 13-year-old boy (a), an 11year-old girl (b) and a 10-year-old girl (c). All bowel CEUS examinations were performed on the same US unit and the log-converted time-intensity curves were generated off-line using the same quantification contrast software. Regions of interest were placed within the thickened bowel wall, which was imaged in longitudinal plane. The vertical axis of the time-intensity curves graph represents the signal intensity of contrast enhancement expressed in a logarithmic scale. The horizontal axis represents the time interval (in seconds) since the administration of contrast agent, which occurs at $0 \mathrm{~s}$. The height of the upward slope (solid arrows) represents the peak enhancement. The area under the curve (open arrows) corresponds to the regional blood volume. There is a progressive increase of the peak enhancement and also of the area under the curve in mild (a), moderate (b) and severe (c) grades of inflammatory disease. Peak enhancement and area under the curve can provide an objective measurement for treatment monitoring 
Fig. 3 Anti-tumor necrosis factor alpha (anti-TNF $\alpha$ ) treatment in a 14-year-old boy with known Crohn disease. Sigmoid colon stenosis was detected on routine US examination and later confirmed on colonoscopy. Treatment with a corticosteroid was initiated. a, b Contrast-enhanced ultrasound (CEUS) examination of this bowel segment was performed to evaluate the activity of the inflammation. Dual image with simultaneous side-by-side display of the contrastenhanced (left) and gray-scale (right) modes. Longitudinal views. CEUS at the initial presentation (a). The stenotic bowel segment (arrowheads) and the pre-stenotic dilation (arrows) are clearly demonstrated. The stenotic segment appears thickened and demonstrates moderate homogeneous wall enhancement. Mild enhancement of the submucosal layer in the prestenotic segment is also noted. Follow-up CEUS 3 weeks after the initiation of corticosteroid treatment (b). There is significant improvement of the enhancement in the stenotic and pre-stenotic segments, indicative of a good response to the treatment. No more signs of active inflammation are noted
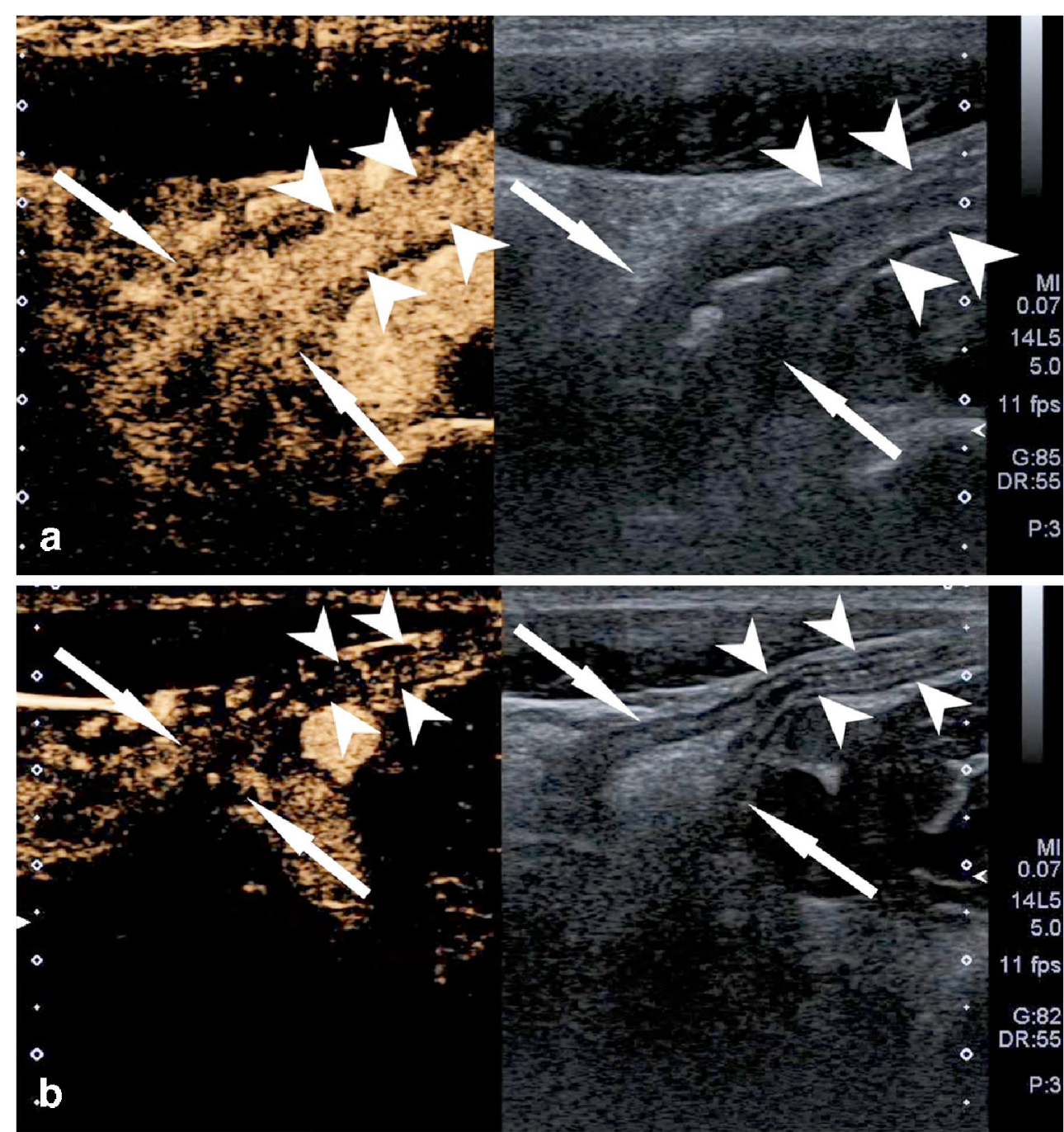

potential of CEUS to accurately detect active inflammation and to correctly discriminate between inflammatory and fibrotic strictures in the pediatric population (Fig. 6) [11].

The third and most recent study compared the performance of CEUS and MRI in 20 children with known or suspected Crohn disease. In this study, Lumason was injected at a bolus dose of $0.03 \mathrm{~mL} / \mathrm{kg}$ and qualitative evaluation of the distal small bowel was performed to assess the enhancement of the bowel wall and pericolonic fat. Considering the MRI examination to be the gold standard imaging modality, the sensitivity of CEUS to detect bowel inflammation was $100 \%$. In $85 \%$ of children (17/20 children) there was agreement between CEUS and MRI findings regarding the presence or absence of enhancement. In the remaining $15 \%$ (3/20 children), CEUS revealed marked bowel inflammation in one child in whom MRI showed only minimal bowel wall enhancement, whereas in two children CEUS was the only modality to reveal intense pericolonic inflammation, thereby facilitating the diagnosis of Crohn disease. The results of this small feasibility and reliability study support the role of CEUS in assessing for small bowel inflammation in pediatric patients with Crohn disease.

\section{Other inflammatory bowel applications in children}

Contrast-enhanced ultrasound of the bowel can also be used in children as a targeted examination to provide valuable added information to answer a specific clinical question, such as in equivocal cases of appendicitis. Appendicitis is a very common cause of acute abdominal pain, with a reported incidence of 9.4-11.0 per 10,000 person-years [63]. Appendicitis can be adequately diagnosed with gray-scale US. However, while positive US findings have a relatively high positive predictive value, identifying the appendix is occasionally difficult. In equivocal or indeterminate cases, MRI or CT is often performed. In specific clinical settings when a child has a contraindication or 

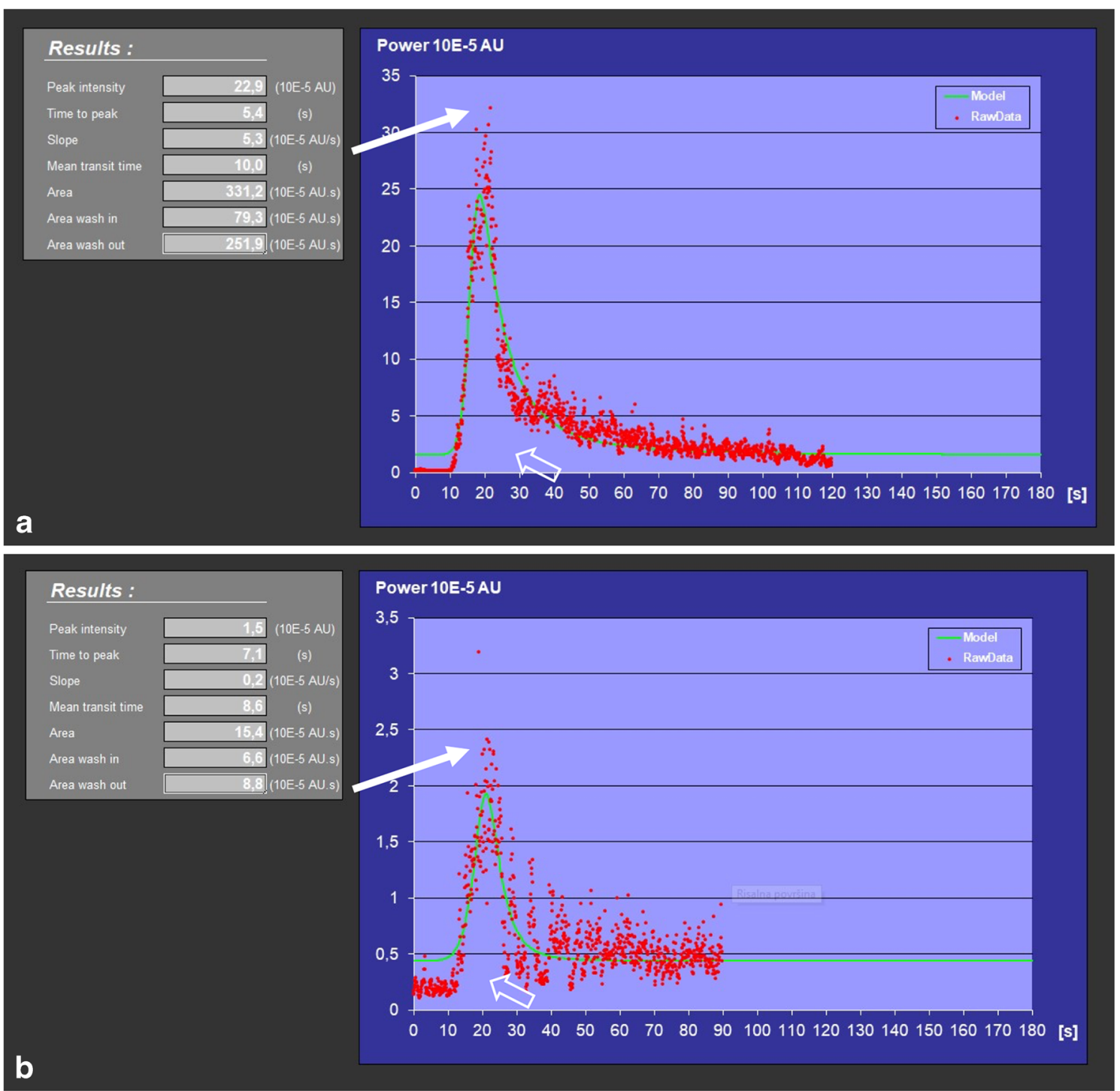

Fig. 4 Inadequate response to azathioprine and enteral diet in a 13-yearold boy with relapse of Crohn disease. Change of therapy was decided. a, b Quantitative contrast-enhanced ultrasound (CEUS) of the bowel with time-intensity curve analysis was performed before (a) and after (b) two cycles of anti-tumor necrosis factor alpha (anti-TNF $\alpha$, infliximab) therapy. Regions of interest were placed in the thickened bowel wall, which was imaged in longitudinal plane. The vertical axis of the graph represents the signal intensity and the horizontal axis of the graph represents the time interval (in seconds) since the injection of the contrast medium that was performed at $0 \mathrm{~s}$. The height of the upward slope (solid arrows) represents the peak enhancement. The area under the curve (open arrows) corresponds to the regional blood flow and volume. There is considerable decrease in the height of the upward slope and in the area under the curve, indicative of good response to treatment. Note the units in the longitudinal axis on the initial study are $\times 10$ higher

without internal vascularity and often surrounded by a hypoechoic rim. CEUS can confirm epiploic appendagitis by showing a mixed enhancement pattern of the mesenteric mass, with variable degrees of peripheral hyperenhancing tissue and a small central non-enhancing region (Fig. 8) [7, 28].

Other reported applications of CEUS include the evaluation of bowel wall or mesenteric neoplastic and inflammatory masses, including their differentiation from colonic content [64]. However, there are no reported applications for the assessment of juvenile polyps (hamartomas). 
Fig. 5 A fulminant course of Crohn disease in a 10-year-old girl. a, b Longitudinal contrastenhanced ultrasound (CEUS) examination of the sigmoid and descending colon. Dual image with simultaneous side-by-side display of the contrast-enhanced (left) and gray-scale (right) modes. CEUS examination performed at the time of diagnosis before introduction of anti-TNF $\alpha$ medication treatment (a). There is markedly thickened bowel wall with intense transmural hyperenhancement and surrounding mesenteric enhancement indicative of severe disease activity. Time-intensity curve demonstrates high values for peak enhancement and area under the curve. Follow-up CEUS examination 2.5 months later (b) demonstrates similar findings as the baseline CEUS examination, indicating no significant response to therapy
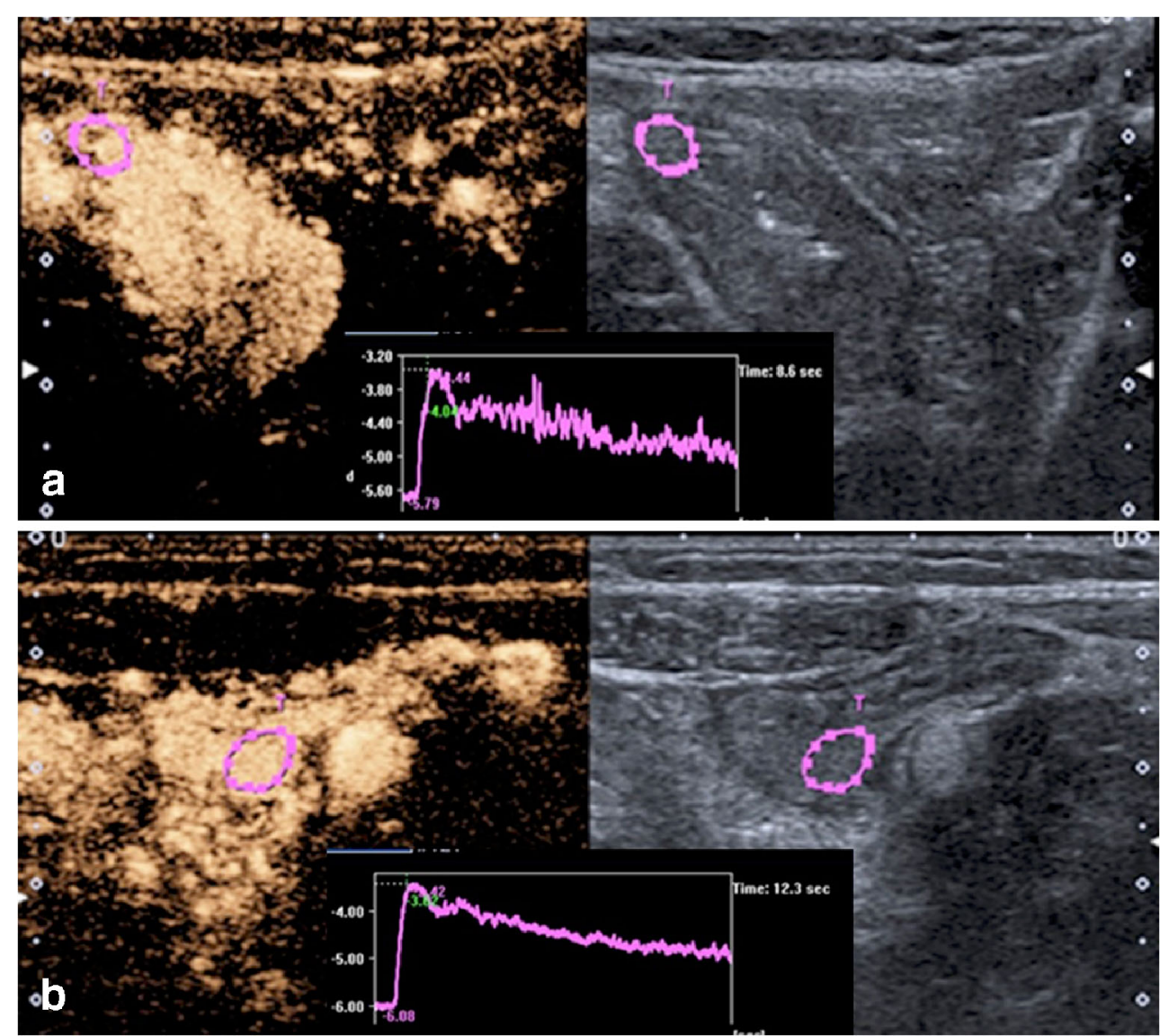

\section{Evaluation of bowel wall perfusion in children}

There is increasing exploration of the role of CEUS to evaluate pediatric bowel perfusion. Although its use is determined on a case-by-case approach, there are specific clinical settings where bowel CEUS can play an important adjunct or problemsolving role. Necrotizing enterocolitis is a potentially lifethreatening condition characterized initially by bowel wall

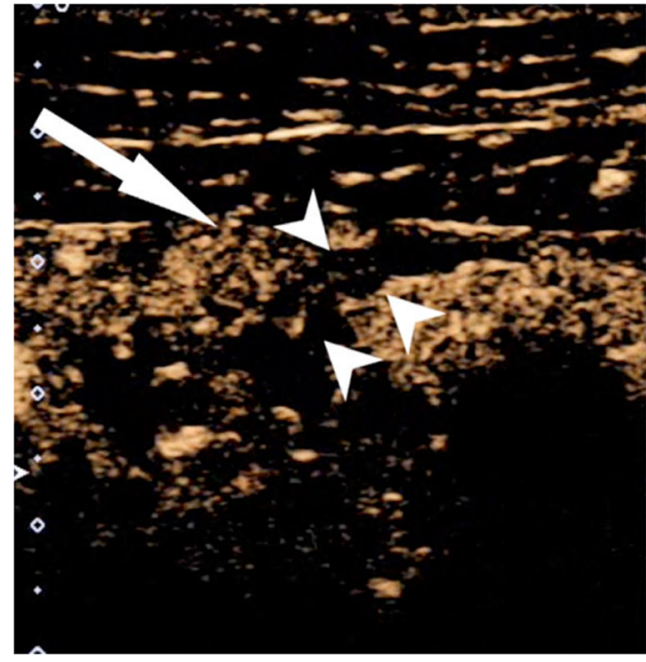

Fig. 6 Crohn disease of the terminal ileum in a 12-year-old girl. Longitudinal contrast-enhanced ultrasound (CEUS) examination of the terminal ileum, dual image with simultaneous side-by-side display of the contrast-enhanced (left) and gray-scale (right) modes. Approximately $10 \mathrm{~cm}$ from the ileocecal valve, there is a short segment of bowel stenosis (arrowheads) with pre-stenotic dilatation. CEUS shows a mild

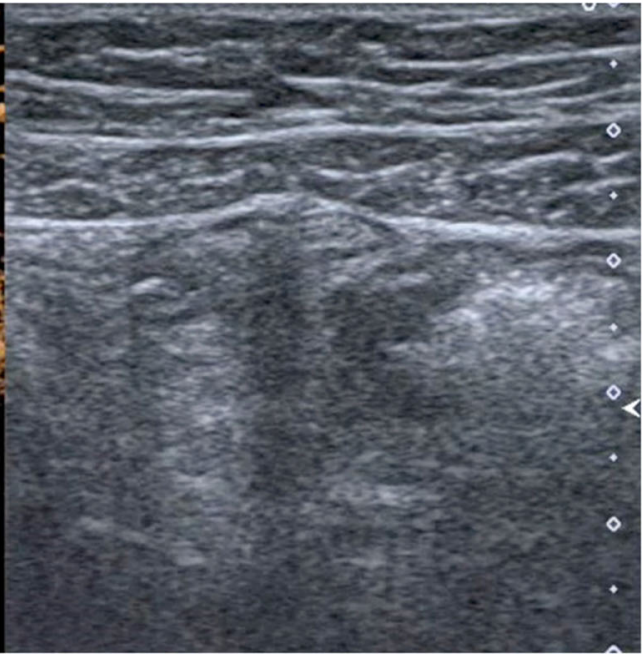

degree of enhancement of the bowel wall near stenosis, but the stenotic part is not enhancing, presumably because of dominant fibrosis. In addition, next to the stenotic segment, another small bowel segment (arrow) is closely opposed and remained attached to the stenotic segment throughout the CEUS examination. This was a highly suspicious sign for presence of entero-enteric fistula 


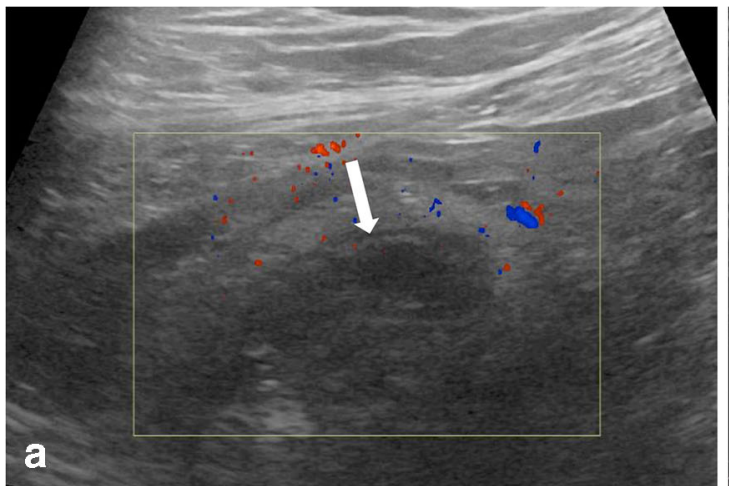

Fig. 7 Contrast-enhanced ultrasound (CEUS) in an 8-year-old boy who presented with right lower quadrant pain and diagnosis of appendicitis. a Color Doppler US image in longitudinal plane demonstrates the blindending tip of the appendix (arrow), which is dilated with a thick wall but shows no significant hyperemia. There is increased echogenicity of the surrounding mesentery. Apart from the lack of pronounced wall hyperemia, the combination of the other findings is radiologically suggestive of appendicitis. b CEUS of the appendix was subsequently

inflammation followed by ischemia that leads to increased permeability of the bowel wall to bacteria. Necrotizing enterocolitis can develop within the first 10 days of age, most commonly at 2-3 days after birth [65]. US is used for these children as a complementary imaging modality to radiography to monitor for complications, including bowel perforation. In the inflammatory phases, the bowel wall is thickened and hyperemic, and it can progress at variable rates to bowel wall thinning and ischemia [66]. Doppler US assessment of the bowel can be challenging in infants who require mechanical ventilation, especially high-frequency oscillation, because of the transmitted vibrations from the ventilator to the body. In this performed to increase the diagnostic confidence. Longitudinal dual image with simultaneous side-by-side display of the gray-scale (left) and contrast-enhanced (right) modes. The appendix demonstrates wall thickening and dilation, as well as significant hyperenhancement (arrow), with no surrounding fluid collection. There is also hyperenhancement of the surrounding inflammed mesenteric fat. Subsequent appendectomy confirmed acute suppurative non-perforated appendicitis with serositis and periappendicitis

setting, CEUS can be used to evaluate the bowel wall perfusion to assess prognosis and to direct care. Depending on the necrotizing enterocolitis phase, CEUS findings initially demonstrate hyperenhancement as the bowel wall becomes inflamed, then progress to hypoenhancement as the bowel wall vasculature is compromised, and finally show no perceptible enhancement within the bowel wall as ischemic changes occur; surrounding structures continue to enhance normally (Online Supplementary Material 3) [67].

Because CEUS is more sensitive to low volume and slow rate of blood flow compared with Doppler US, it could also be useful in children with complicated intussusception. Here it

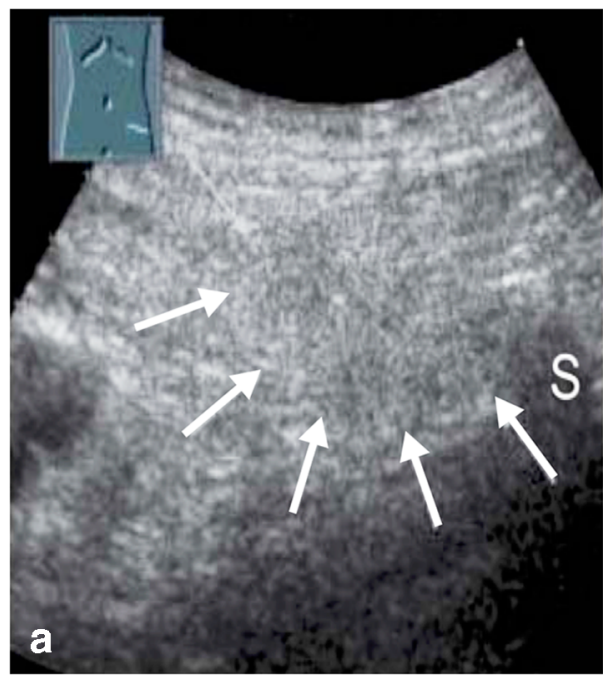

Fig. 8 Acute epiploic appendagitis in a 78-year-old man. a Gray-scale US of the sigmoid colon in transverse plane shows a homogeneous hyperechoic mesenteric mass (arrows) located near the sigmoid colon $(S)$. b Contrast-enhanced ultrasound (CEUS) in transverse plane shows mixed enhancement of the mesenteric mass with a central non-enhancing region surrounded by an oval region of hyperenhancement (arrows). c

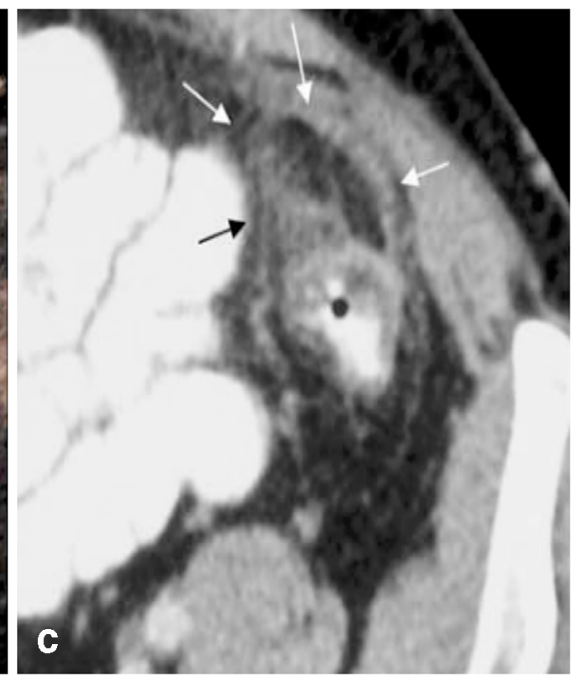

Axial CT shows a fat-density lesion (arrows) adjacent to the sigmoid colon, demonstrating a central hyperdense dot and surrounded by a thin high-density rim. There is inflammatory stranding of the associated mesentery and thickening of the adjacent peritoneal lining. Imaging findings are in keeping with epiploic appendagitis. Reprinted from [7] with permission 

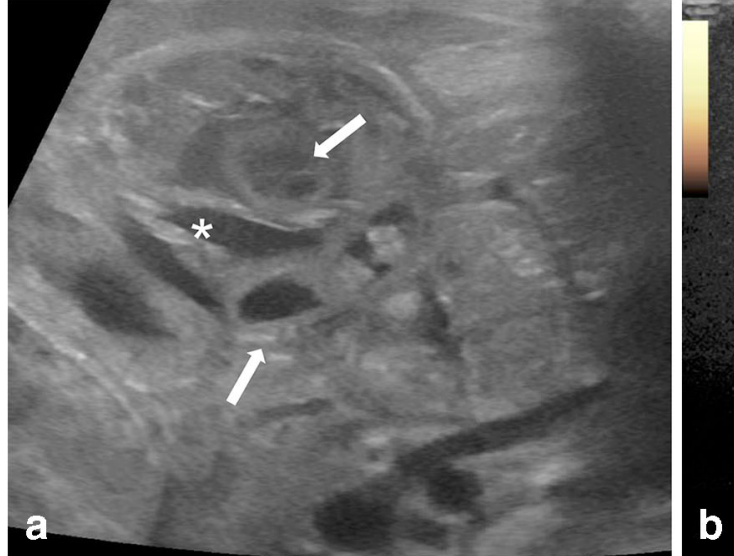

Fig. 9 Preterm 1-day-old girl who presented with concern for duodenal atresia based on a prenatal US that was performed to evaluate for bowel perfusion because of theorized in utero volvulus. a Transverse postnatal baseline US scan shows a cluster of featureless echogenic aperistaltic bowel loops (arrows) without significant wall thickening. There is a small amount of free fluid with mild complexity manifested as several septations and echoes (asterisk). There was a normal orientation of the superior mesenteric artery and vein. b Transverse postnatal contrastenhanced ultrasound (CEUS). Dual image with simultaneous side-byside display of the gray-scale (left) and contrast-enhanced (right)

could serve as a quick method for evaluating mural perfusion at the time of initial diagnosis, prior to US/fluoroscopic reduction or surgical intervention, improving the color Doppler assessment [8].

In the intensive care unit or emergency setting, there are cases requiring evaluation of bowel perfusion. At our institution, CEUS evaluation for bowel perfusion has been performed as a follow-up to possible duodenal atresia or in utero volvulus seen on fetal sonogram (Fig. 9).

\section{Strengths and limitations}

While diagnostic images of the bowel can be obtained with CEUS in most pediatric patients, there are occasional challenges and limitations. Several of these difficulties are no different from those associated with gray-scale and Doppler bowel US, including difficulty in visualizing the bowel segment of interest because of body habitus, deep location of bowel segments of interest within the abdomen or pelvis, or excessive overlying bowel gas $[29,30]$. Occasionally, graded compression of the bowel with the transducer, similar to the technique commonly used to identify the appendix, can displace the overlying bowel gas and improve bowel wall visualization. Keep in mind that additional pressure can affect bowel wall enhancement and time-intensity curve results, so applying steady pressure during quantitative analysis is suggested. Deep bowel loops might be better visualized using a lower transducer frequency or a different transducer (i.e., curved as opposed to linear). Imaging with a full urinary bladder can lift small bowel loops located in the deep pelvis into a more favorable superficial position [29, 30].

Quantitative CEUS for intestinal perfusion has other distinct limitations. First, peristalsis or respiratory motion can make it difficult to keep an ROI consistently within a segment of the bowel wall to create a time-intensity curve, and accurate quantitative assessment of the perfusion (Online Supplementary Material 4). Fortuitously, many bowel segments affected by Crohn disease have diminished motility and so they are relatively fixed in position, leading to successful quantification [1-3]. Second, inter-operator agreement could affect comparison of one examination to another because of multiple factors, including the UCA dose and administration rate, the size of the intravenous catheter, as well as location and size of the ROI over the affected bowel wall. Last, there are inconsistent reports on correlation of perfusion quantitative parameters with clinical, endoscopic or surgical findings. A standardized methodology might help overcome this limitation and allow for more uniform interpretation and outcomes research.

\section{Conclusion}

Contrast-enhanced ultrasound applications in pediatric bowel are evolving as the increased sensitivity to blood flow detection proves advantageous over US for the assessment of bowel perfusion. IBD is the main CEUS application for bowel in children and adults. However, there is a role for CEUS as a problem-solving tool in other bowel conditions. Expanding knowledge of bowel CEUS techniques, including improved 
and standardized quantification methods, will enable radiologists to embrace this examination as a primary or adjunct tool for assessing the bowel.

\section{Compliance with ethical standards}

Conflicts of interest Dr. Dillman receives grant funding from Bracco Diagnostics.

\section{References}

1. Medellin A, Merrill C, Wilson SR (2018) Role of contrastenhanced ultrasound in evaluation of the bowel. Abdom Radiol 43:918-933

2. Pecere S, Holleran G, Ainora ME et al (2018) Usefulness of contrast-enhanced ultrasound (CEUS) in inflammatory bowel disease (IBD). Dig Liver Dis 50:761-767

3. Ripolles T, Martinez-Perez MJ, Blanc E et al (2011) Contrastenhanced ultrasound (CEUS) in Crohn's disease: technique, image interpretation and clinical applications. Insights Imaging 2:639-652

4. Serafin Z, Bialecki M, Bialecka A et al (2016) Contrast-enhanced ultrasound for detection of Crohn's disease activity: systematic review and meta-analysis. J Crohns Colitis 10:354-362

5. Ma X, Li Y, Jia H et al (2015) Contrast-enhanced ultrasound in the diagnosis of patients suspected of having active Crohn's disease: meta-analysis. Ultrasound Med Biol 41:659-668

6. Incesu L, Yazicioglu AK, Selcuk MB, Ozen N (2004) Contrastenhanced power Doppler US in the diagnosis of acute appendicitis. Eur J Radiol 50:201-209

7. Gorg C, Egbring J, Bert T (2009) Contrast-enhanced ultrasound of epiploic appendagitis. Ultraschall Med 30:163-167

8. Rafailidis V, Phillips C, Yusuf G, Sidhu P (2017) A case of adult intussusception with greyscale, contrast-enhanced ultrasound and computerised tomography correlation. Ultrasound 25:120-125

9. Mudambi K, Sandberg J, Bass D, Rubesova E (2020) Contrast enhanced ultrasound: comparing a novel modality to MRI to assess for bowel disease in pediatric Crohn's patients. Transl Gastroenterol Hepatol 5:13

10. Benjamin JL, Dennis R, White S Jr et al (2020) Improved diagnostic sensitivity of bowel disease of prematurity on contrast-enhanced ultrasound. J Ultrasound Med 39:1031-1036

11. Thimm MA, Cuffari C, Garcia A et al (2019) Contrast-enhanced ultrasound and shear wave elastography evaluation of Crohn's disease activity in three adolescent patients. Pediatr Gastroenterol Hepatol Nutr 22:282-290

12. Kljucevsek D, Vidmar D, Urlep D, Dezman R (2016) Dynamic contrast-enhanced ultrasound of the bowel wall with quantitative assessment of Crohn's disease activity in childhood. Radiol Oncol 50:347-354

13. Zezos P, Zittan E, Islam S et al (2019) Associations between quantitative evaluation of bowel wall microvascular flow by contrastenhanced ultrasound and indices of disease activity in Crohn's disease patients using both bolus and infusion techniques. J Clin Ultrasound 47:453-460

14. Martinez MJ, Ripolles T, Paredes JM et al (2019) Intravenous contrast-enhanced ultrasound for assessing and grading postoperative recurrence of Crohn's disease. Dig Dis Sci 64:1640-1650

15. Wilkens R, Hagemann-Madsen RH, Peters DA et al (2018) Validity of contrast-enhanced ultrasonography and dynamic contrast-enhanced MR enterography in the assessment of transmural activity and fibrosis in Crohn's disease. J Crohns Colitis 12:48-56
16. Goertz RS, Klett D, Wildner D et al (2018) Quantitative contrastenhanced ultrasound for monitoring vedolizumab therapy in inflammatory bowel disease patients: a pilot study. Acta Radiol 59: $1149-1156$

17. Giannetti A, Matergi M, Biscontri M, Franci L (2017) Contrastenhanced ultrasonography in clinical practice in Crohn's disease: feasibility of destruction/replenishment method and software analysis. J Ultrasound 20:309-319

18. Coelho R, Ribeiro H, Maconi G (2017) Bowel thickening in Crohn's disease: fibrosis or inflammation? Diagnostic ultrasound imaging tools. Inflamm Bowel Dis 23:23-34

19. Quaia E, Cabibbo B, De Paoli L et al (2013) The value of timeintensity curves obtained after microbubble contrast agent injection to discriminate responders from non-responders to antiinflammatory medication among patients with Crohn's disease. Eur Radiol 23:1650-1659

20. Liu C, Xu XR, Xu HX et al (2015) Conventional ultrasound and contrast-enhanced ultrasound in evaluating the severity of Crohn's disease. Int J Clin Exp Med 8:123-134

21. Nylund K, Jirik R, Mezl M et al (2013) Quantitative contrastenhanced ultrasound comparison between inflammatory and fibrotic lesions in patients with Crohn's disease. Ultrasound Med Biol 39: 1197-1206

22. Romanini L, Passamonti M, Navarria M et al (2014) Quantitative analysis of contrast-enhanced ultrasonography of the bowel wall can predict disease activity in inflammatory bowel disease. Eur J Radiol 83:1317-1323

23. Saevik F, Nylund K, Hausken T et al (2014) Bowel perfusion measured with dynamic contrast-enhanced ultrasound predicts treatment outcome in patients with Crohn's disease. Inflamm Bowel Dis 20:2029-2037

24. Wong DD, Forbes GM, Zelesco M et al (2012) Crohn's disease activity: quantitative contrast-enhanced ultrasound assessment. Abdom Imaging 37:369-376

25. Ripolles T, Martinez-Perez MJ, Paredes JM et al (2013) Contrastenhanced ultrasound in the differentiation between phlegmon and abscess in Crohn's disease and other abdominal conditions. Eur J Radiol 82:e525-e531

26. Mao R, Chen YJ, Chen BL et al (2018) Intra-cavitary contrastenhanced ultrasound: a novel radiation-free method to detect abscess associated penetrating disease in Crohn's disease. J Crohns Colitis 13:593-599

27. Kljucevsek D, Riccabona M, Ording Muller LS et al (2020) Intracavitary contrast-enhanced ultrasonography in children: review with procedural recommendations and clinical applications from the European Society of Paediatric Radiology abdominal imaging task force. Pediatr Radiol 50:596-606

28. Menozzi G, Maccabruni V, Zanichelli M, Massari M (2014) Contrast-enhanced ultrasound appearance of primary epiploic appendagitis. J Ultrasound 17:75-76

29. Atkinson NSS, Bryant RV, Dong Y et al (2017) How to perform gastrointestinal ultrasound: anatomy and normal findings. World $\mathrm{J}$ Gastroenterol 23:6931-6941

30. Gale HI, Gee MS, Westra SJ, Nimkin K (2016) Abdominal ultrasonography of the pediatric gastrointestinal tract. World J Radiol 8: 656-667

31. Sidhu PS, Cantisani V, Dietrich CF et al (2018) The EFSUMB guidelines and recommendations for the clinical practice of contrast-enhanced ultrasound (CEUS) in non-hepatic applications: update 2017 (long version). Ultraschall Med 39:e2-e44

32. Ripolles T, Martinez-Perez MJ, Paredes JM et al (2019) The role of intravenous contrast agent in the sonographic assessment of Crohn's disease activity: is contrast agent injection necessary? J Crohns Colitis 13:585-592 
33. Wilkens R, Wilson A, Burns PN et al (2018) Persistent enhancement on contrast-enhanced ultrasound studies of severe Crohn's disease: stuck bubbles? Ultrasound Med Biol 44:2189-2198

34. Bialecki M, Bialecka A, Laskowska K et al (2014) Contrastenhanced ultrasonography for the determination of Crohn's disease activity — preliminary experience. Pol J Radiol 79:70-74

35. Ripolles T, Rausell N, Paredes JM et al (2013) Effectiveness of contrast-enhanced ultrasound for characterisation of intestinal inflammation in Crohn's disease: a comparison with surgical histopathology analysis. J Crohns Colitis 7:120-128

36. Quaia E (2013) Contrast-enhanced ultrasound of the small bowel in Crohn's disease. Abdom Imaging 38:1005-1013

37. Paredes JM, Ripolles T, Cortes X et al (2013) Contrast-enhanced ultrasonography: usefulness in the assessment of postoperative recurrence of Crohn's disease. J Crohns Colitis 7:192-201

38. Ripolles T, Martinez MJ, Paredes JM et al (2009) Crohn disease: correlation of findings at contrast-enhanced US with severity at endoscopy. Radiology 253:241-248

39. Migaleddu V, Scanu AM, Quaia E et al (2009) Contrast-enhanced ultrasonographic evaluation of inflammatory activity in Crohn's disease. Gastroenterology 137:43-52

40. Quaia E, Gennari AG, Cova MA, van Beek EJR (2018) Differentiation of inflammatory from fibrotic ileal strictures among patients with Crohn's disease based on visual analysis: feasibility study combining conventional B-mode ultrasound, contrastenhanced ultrasound and strain elastography. Ultrasound Med Biol 44:762-770

41. Braden B, Ignee A, Hocke $M$ et al (2010) Diagnostic value and clinical utility of contrast enhanced ultrasound in intestinal diseases. Dig Liver Dis 42:667-674

42. Serra C, Menozzi G, Labate AM et al (2007) Ultrasound assessment of vascularization of the thickened terminal ileum wall in Crohn's disease patients using a low-mechanical index real-time scanning technique with a second generation ultrasound contrast agent. Eur J Radiol 62:114-121

43. De Pascale A, Garofalo G, Perna M et al (2006) Contrast-enhanced ultrasonography in Crohn's disease. Radiol Med 111:539-550

44. Robotti D, Cammarota T, Debani P et al (2004) Activity of Crohn disease: value of color-power-Doppler and contrast-enhanced ultrasonography. Abdom Imaging 29:648-652

45. Piscaglia F, Nolsoe C, Dietrich CF et al (2012) The EFSUMB guidelines and recommendations on the clinical practice of contrast enhanced ultrasound (CEUS): update 2011 on non-hepatic applications. Ultraschall Med 33:33-59

46. Campani R, Calliada F, Bottinelli O et al (1998) Contrast enhancing agents in ultrasonography: clinical applications. Eur J Radiol 27: S161-S170

47. van Wassenaer EA, de Voogd FAE, van Rijn RR et al (2020) Bowel ultrasound measurements in healthy children - systematic review and meta-analysis. Pediatr Radiol 50:501-508

48. Chiorean L, Schreiber-Dietrich D, Braden B et al (2014) Transabdominal ultrasound for standardized measurement of bowel wall thickness in normal children and those with Crohn's disease. Med Ultrason 16:319-324

49. Quaia E, Migaleddu V, Baratella E et al (2009) The diagnostic value of small bowel wall vascularity after sulfur hexafluoridefilled microbubble injection in patients with Crohn's disease. Correlation with the therapeutic effectiveness of specific antiinflammatory treatment. Eur J Radiol 69:438-444

50. Ponder A, Long MD (2013) A clinical review of recent findings in the epidemiology of inflammatory bowel disease. Clin Epidemiol 5: 237-247
51. Danese S, Fiocchi C (2011) Ulcerative colitis. N Engl J Med 365: 1713-1725

52. Quaia E, De Paoli L, Stocca T et al (2012) The value of small bowel wall contrast enhancement after sulfur hexafluoride-filled microbubble injection to differentiate inflammatory from fibrotic strictures in patients with Crohn's disease. Ultrasound Med Biol 38:1324-1332

53. Di Sabatino A, Fulle I, Ciccocioppo R et al (2002) Doppler enhancement after intravenous Levovist injection in Crohn's disease. Inflamm Bowel Dis 8:251-257

54. Girlich C, Jung EM, Huber E et al (2011) Comparison between preoperative quantitative assessment of bowel wall vascularization by contrast-enhanced ultrasound and operative macroscopic findings and results of histopathological scoring in Crohn's disease. Ultraschall Med 32:154-159

55. Schreyer AG, Finkenzeller T, Gossmann H et al (2008) Microcirculation and perfusion with contrast enhanced ultrasound (CEUS) in Crohn's disease: first results with linear contrast harmonic imaging (CHI). Clin Hemorheol Microcirc 40:143-155

56. Mansuri I, Fletcher JG, Bruining DH et al (2017) Endoscopic skipping of the terminal ileum in pediatric Crohn disease. AJR Am J Roentgenol 208:W216-W224

57. Dillman JR, Rubin JM, Johnson LA et al (2017) Can contrastenhanced sonography detect bowel wall fibrosis in mixed inflammatory and fibrotic Crohn disease lesions in an animal model? J Ultrasound Med 36:523-530

58. Abraham BP, Mehta S, El-Serag HB (2012) Natural history of pediatric-onset inflammatory bowel disease: a systematic review. J Clin Gastroenterol 46:581-589

59. Rosen MJ, Dhawan A, Saeed SA (2015) Inflammatory bowel disease in children and adolescents. JAMA Pediatr 169:1053-1060

60. Benchimol EI, Fortinsky KJ, Gozdyra P et al (2011) Epidemiology of pediatric inflammatory bowel disease: a systematic review of international trends. Inflamm Bowel Dis 17:423-439

61. Oliveira SB, Monteiro IM (2017) Diagnosis and management of inflammatory bowel disease in children. BMJ 357:j2083

62. Gasparetto M, Guariso G, Pozza LV et al (2016) Clinical course and outcomes of diagnosing inflammatory bowel disease in children 10 years and under: retrospective cohort study from two tertiary centres in the United Kingdom and in Italy. BMC Gastroenterol 16:35

63. Horn CB, Tian D, Bochicchio GV, Turnbull IR (2018) Incidence, demographics, and outcomes of nonoperative management of appendicitis in the United States. J Surg Res 223:251-258

64. Zhang F, Miao LY, Ge HY et al (2018) Usefulness of contrastenhanced ultrasound in differentiating inflammatory bowel disease from colon cancer. Ultrasound Med Biol 44:124-133

65. Lin PW, Stoll BJ (2006) Necrotising enterocolitis. Lancet 368: 1271-1283

66. Epelman M, Daneman A, Navarro OM et al (2007) Necrotizing enterocolitis: review of state-of-the-art imaging findings with pathologic correlation. Radiographics 27:285-305

67. Al-Hamad S, Hackam DJ, Goldstein SD et al (2018) Contrastenhanced ultrasound and near-infrared spectroscopy of the neonatal bowel: novel, bedside, noninvasive, and radiation-free imaging for early detection of necrotizing enterocolitis. Am J Perinatol 35: $1358-1365$

Publisher's note Springer Nature remains neutral with regard to jurisdictional claims in published maps and institutional affiliations. 